\title{
Stability of intersite dark solitons in a parametrically driven discrete nonlinear Schrödinger equation
}

\author{
O.P. Swami ${ }^{1}$, V. Kumar ${ }^{2}$, B. Suthar ${ }^{3}$, A. K. Nagar $^{2}$ \\ ${ }^{1}$ Department of Physics, Government College, Loonkaransar, Bikaner, Rajasthan, 334603, India \\ ${ }^{2}$ Department of Physics, Government Dungar College, Bikaner, Rajasthan, 334001, India \\ ${ }^{3}$ Department of Physics, MLB Government College, Nokha, Bikaner, Rajasthan, 334803, India \\ omg1789@gmail.com, vijendrasaini2009@gmail.com, bhuvneshwer@gmail.com, ajaya.nagar@gmail.com
}

PACS 05.45.Yv, 42.65.Tg, 63.20.Pw, 11.15.Bt

DOI 10.17586/2220-8054-2019-10-4-391-397

In this paper, a parametrically driven discrete nonlinear Schrödinger equation will be considered for defocusing case. Analytical and numerical calculations will be performed to determine the existence and stability of intersite dark discrete solitons admitted by discrete nonlinear Schrödinger equation. It will be shown that a parametric driving can stabilizes intersite discrete dark solitons. Stability windows of all the fundamental solitons will be presented and approximations to the onset of instability will be derived using perturbation theory, with accompanying numerical results.

Keywords: soliton, discrete nonlinear Schrödinger equation, parametrically driven, instability, perturbation theory.

Received: 4 May 2019

Revised: 26 May 2019

\section{Introduction}

We consider a parametrically driven discrete nonlinear Schrödinger (PDNLS) equation for defocusing case:

$$
i \dot{\varphi}_{n}=-C \Delta_{2} \varphi_{n}+\left|\varphi_{n}\right|^{2} \varphi_{n}-\omega \varphi_{n}-\gamma \bar{\varphi}_{n},
$$

where, $\varphi_{n} \equiv \varphi_{n}(t)$ is a real-valued wave function at site $n$, the overdot and the overline indicates to time derivative and complex conjugation, respectively. The coupling constant between two adjacent sites is represented by $C . \Delta_{2} \varphi_{n}=$ $\varphi_{n+1}-2 \varphi_{n}+\varphi_{n-1}$ is the one-dimensional (1D) discrete Laplacian, $\gamma$ is the parametric driving coefficient with frequency $\omega$. In the absence of parametric driving, i.e., for $\gamma=0$, Eq. (1) is reduced to the standard discrete nonlinear Schrödinger (DNLS) equation which appears in a wide range of important applications [1-5] and admits dark solitons with defocusing nonlinearities, respectively.

With a focusing nonlinearity and finite $C$ PDNLS has been studied first by Susanto et al. [6,7,9]. The PDNLS model was also derived for coupled arrays of nonlinear resonators in micro and nano-electromechanical systems [8,10]. The same equation also applies to the study of Bose-Einstein condensates and long bosonic Josephson junctions [11, 12]. Dynamics of the parametrically driven NLS solitons beyond the onset of the oscillatory instability was studied by N. V. Alexeeva et al. [13]. Bright and dark solitons in optical media with intensity-dependent non-linear response as well as two-component solitary waves supported by parametric wave mixing in quadratic or cubic media was studied by Yu. S. Kivshar [14]. Theory of stationary solitary waves generated by optical parametric interactions in the presence of Kerr-type nonlinearities and dissipations was studied by K. Hayata and M. Koshiba [15].

In this study, the existence and stability of the fundamental intersite dark solitons in the defocusing PDNLS is examined analytically and numerically. The method of perturbation theory for small $C$ is adopted in analytical calculation. Numerical comparisons are made by solving a corresponding eigenvalue problem.

Stationary solution of system (1) is in the form of $\varphi_{n}=Z_{n}$, where $Z_{n}$ is a real-valued and time independent, satisfy the following stationary equation:

$$
-C \Delta_{2} Z_{n}+Z_{n}^{3}-\omega Z_{n}-\gamma Z_{n}=0
$$

The solution of Eq. (1) is the form of solitonic and its linear stability is determined by solving a corresponding eigenvalue problem. A linearization ansatz is introduced as follows

$$
\varphi_{n}=Z_{n}+\delta \rho_{n},
$$

where $\delta \ll 1$, and substitute this into the defocusing Eq. (1), to yield the following linearized equation at $O(\delta)$ :

$$
i \dot{\rho}_{n}=-C \Delta_{2} \rho_{n}-2\left|Z_{n}\right|^{2} \rho_{n}-Z_{n}^{2} \bar{\rho}_{n}+\omega \rho_{n}+\gamma \bar{\rho}_{n}
$$


by writing $\rho_{n}=\eta_{n}+i \zeta_{n}$, equation (3) can be transformed into the eigenvalue problem:

$$
\left(\begin{array}{c}
\dot{\eta}_{n} \\
\dot{\zeta}_{n}
\end{array}\right)=M\left(\begin{array}{c}
\eta_{n} \\
\zeta_{n}
\end{array}\right),
$$

where:

$$
M=\left(\begin{array}{cc}
0 & L_{+}(C) \\
L_{-}(C) & 0
\end{array}\right),
$$

and the operators $L_{+}(C)$ and $L_{-}(C)$ are defined by

$$
\begin{aligned}
& L_{+}(C) \equiv-C \Delta_{2}+\left(3 Z_{n}^{2}-\omega-\gamma\right), \\
& L_{-}(C) \equiv-C \Delta_{2}-\left(Z_{n}^{2}-\omega+\gamma\right) .
\end{aligned}
$$

By the eigenvalues of $M$ the stability of $Z_{n}$ is determined. Let the eigenvalues of $M$ is denoted by $i d$, which implies that $Z_{n}$ is stable if $\operatorname{Im}(d)=0$. Because the Eq. (5) is linear then one of the 'eigenvectors', for instance $\zeta_{n}$, can be eliminated, from which following eigenvalue problem is obtained

$$
L_{+}(C) L_{-}(C) \eta_{n} \equiv \mathrm{d}^{2} \eta_{n}=\Omega \eta_{n} .
$$

\section{Analytical calculations}

To perform analytical calculations of the existence and stability of the intersite dark solitons for small coupling constant $C$, a perturbation analysis is used. By expanding $Z_{n}$ in powers of $C$, the $O(1)$ and $O(C)$ equations are obtained, which give the solutions $Z_{n}$ at order $C$ as:

$$
Z_{n}^{(1)}=\frac{\Delta_{2} Z_{n}^{(0)}}{3\left(Z_{n}^{(0)}\right)^{2}-(\omega+\gamma)} .
$$

Using a perturbative expansion, the configuration of intersite dark soliton in $\mathrm{AC}$ limit $C=0$ is given by:

$$
Z_{n}^{(0)}= \begin{cases}-\sqrt{\omega+\gamma}, & n=0,-1, \ldots \\ \sqrt{\omega+\gamma}, & n=1,2, \ldots\end{cases}
$$

To study the eigenvalue(s) analytically, $\eta_{n}$ and $\omega$ are expanded in power of $C$ as

$$
\begin{aligned}
& \eta_{n}=\eta_{n}^{(0)}+C \eta_{n}^{(1)}+O\left(C^{2}\right), \\
& \omega=\omega^{(0)}+C \omega^{(1)}+O\left(C^{2}\right) .
\end{aligned}
$$

Then equation (6) can be written at $O(1)$ and $O(C)$ as

$$
\left[L_{+}(0) L_{-}(0)-\Omega^{(0)}\right] \eta_{n}^{(0)}=0,
$$

and

where

$$
\left[L_{+}(0) L_{-}(0)-\Omega^{(0)}\right] \eta_{n}^{(1)}=f_{n}
$$

$$
f_{n}=\left[\left(\Delta_{2}-2 Z_{n}^{(0)} Z_{n}^{(1)}\right) L_{-}(0)+L_{+}(0)\left(\Delta_{2}-6 Z_{n}^{(0)} Z_{n}^{(1)}\right)+\Omega^{(1)}\right] \eta_{n}^{(0)} .
$$

To perform a stability analysis, firstly the significance of continuous spectrum for a intersite dark soliton is investigated by substituting a plane-wave expansion:

$$
\eta_{n}=a e^{i k n}+b e^{-i k n} .
$$

From this, the dispersion relation for an intersite dark soliton is obtained as

$$
\Omega=[2 C(\cos k-1)-(\omega+2 \gamma)]^{2}-\omega^{2},
$$

thus the continuous band lies between

when $k=0$, and

$$
\Omega_{L}=4(\omega+\gamma) \gamma
$$

$$
\Omega_{U}=4(\omega+\gamma) \gamma+8 C(\omega+2 \gamma)+O\left(C^{2}\right),
$$

when $k=\pi$. 
It is shown from above analysis that there are only three possibilities for parameter values $\gamma$ and $\omega$ in the continuous spectrum above, i.e., either $\omega \geq \gamma>0, \gamma>\omega>0$ or $\gamma>-\omega \geq 0$. However, this paper is restricted to the first case only.

In this case, the leading order solution is given by the configuration (8) and the next correction can be obtained from Eq. (7) resulting

$$
u_{n}^{(1)}= \begin{cases}0, & n=-2,-3, \ldots \\ \frac{1}{\sqrt{\omega+\gamma}}, & n=-1 \\ -\frac{1}{\sqrt{\omega+\gamma}}, & n=0 \\ 0, & n=1,2, \ldots\end{cases}
$$

Thus the intersite discrete dark soliton is given, with errors of $O\left(C^{2}\right)$, by

$$
u_{n}= \begin{cases}-\sqrt{\omega+\gamma}, & n=-2,-3, \ldots \\ -\sqrt{\omega+\gamma}+\frac{C}{\sqrt{\omega+\gamma}}, & n=-1, \\ \sqrt{\omega+\gamma}-\frac{C}{\sqrt{\omega+\gamma}}, & n=0, \\ \sqrt{\omega+\gamma}, & n=1,2, \ldots\end{cases}
$$

and for this configuration Eq. (6) can be written as

$$
L_{+}(0) L_{-}(0)=4 \omega \gamma+4 \gamma^{2} \text { for all } n,
$$

from which it is concluded that there is a single leading order eigenvalue, given by $\Omega^{(0)}=4 \omega \gamma+4 \gamma^{2}$, with infinite multiplicity. Then a continuous spectrum for nonzero $C$ is formed by the expansion of that eigenvalue.

From the fact of a single eigenvalue above, nothing can be said about the discrete eigenvalue(s) yet. However, its continuation still can be calculated as the coupling constant is turned on, which can be carried out from Eq. (12). Because

$$
L_{+}(0) L_{-}(0)-\Omega^{(0)}=0 \text { for all } n,
$$

the solvability condition for Eq. (12) has required $f_{n}=0$ for all $n$. Then after a simple calculation:

$$
f_{n}= \begin{cases}{\left[4 \omega+16 \gamma+2(\omega+2 \gamma) \Delta_{2}+\Omega^{(1)}\right] \eta_{n}^{(0)},} & n=-1,0 \\ {\left[2(\omega+2 \gamma) \Delta_{2}+\Omega^{(1)}\right] \eta_{n}^{(0)},} & n \neq-1,0\end{cases}
$$

Notice that the eigenvector for a localized mode in which there is only one eigenvalue with infinite multiplicity is also localized and decaying exponentially (see, e.g., Baesens et al. [16] for a justification). Thus, a suitable ansatz for $\eta_{n}^{(0)}$ can be chosen as

$$
\eta_{n}^{(0)}= \begin{cases}P e^{c(n+1.5)}, & n \leq-2 \\ P_{0}, & n=-1 \\ Q_{0}, & n=0 \\ Q e^{-c(n+1.5)}, & n \geq 1\end{cases}
$$

for $P, P_{0}, Q, Q_{0} \neq 0$ and $c>0$. Substituting Eq. (23) in Eq. (22) and after applying solvability condition $f_{n}=0$ for all $n$, following Eqs. are obtained:

$$
\begin{aligned}
P e^{-0.5 c}\left[2(\omega+2 \gamma)\left(e^{-c}-2\right)+\Omega^{(1)}\right]+2 P_{0}(\omega+2 \gamma) & =0, & & n=-2, \\
P_{0}\left(8 \gamma+\Omega^{(1)}\right)+2 Q_{0}(\omega+2 \gamma)+2 P(\omega+2 \gamma) e^{-0.5 c} & =0, & & n=-1, \\
Q_{0}\left(8 \gamma+\Omega^{(1)}\right)+2 P_{0}(\omega+2 \gamma)+2 Q(\omega+2 \gamma) e^{-2.5 c} & =0, & & n=0, \\
Q e^{-2.5 c}\left[2(\omega+2 \gamma)\left(e^{-c}-2\right)+\Omega^{(1)}\right]+2 Q_{0}(\omega+2 \gamma) & =0, & & n=1, \\
2(\omega+2 \gamma)\left(e^{c}-2+e^{-c}\right)+\Omega^{(1)} & =0, & & n \neq-2,-1,1,0 .
\end{aligned}
$$


It is clear from above first four equations that either $P_{0}=Q_{0}$ or $P_{0}=-Q_{0}$. From which it is obtained respectively, either

or

$$
c=\ln \left(\frac{3 \omega+10 \gamma}{\omega+2 \gamma}\right), \quad \Omega^{(1)}=\frac{-8(\omega+4 \gamma)^{2}}{3 \omega+10 \gamma},
$$

$$
c=\ln \left(\frac{\omega+6 \gamma}{\omega+2 \gamma}\right), \quad \Omega^{(1)}=\frac{-32 \gamma^{2}}{\omega+6 \gamma} .
$$

Therefore, the bifurcating discrete eigenvalues of an intersite dark soliton for small $C$ are respectively given by

$$
\begin{gathered}
\Omega_{E, 1}=4 \gamma \omega+4 \gamma^{2}-\frac{8(\omega+4 \gamma)^{2}}{3 \omega+10 \gamma} C+O(C)^{2}, \\
\Omega_{E, 2}=4 \gamma \omega+4 \gamma^{2}-\frac{32 \gamma^{2}}{\omega+6 \gamma} C+O(C)^{2} .
\end{gathered}
$$

In the $\mathrm{AC}$ limit $C=0$, the above eigenvalues correspond, respectively, to the following normalized eigenvectors

$$
\eta_{n}^{(0)}= \begin{cases}\frac{2 \sqrt{(\omega+3 \gamma)(\omega+4 \gamma)}}{\omega+2 \gamma}\left(\frac{3 \omega+10 \gamma}{\omega+2 \gamma}\right)^{n}, & n \leq-2, \\ \frac{2 \sqrt{(\omega+3 \gamma)(\omega+4 \gamma)}}{3 \omega+10 \gamma}, & n=-1, \\ \frac{2 \sqrt{(\omega+3 \gamma)(\omega+4 \gamma)}}{3 \omega+10 \gamma}, & n=0, \\ \frac{2 \sqrt{(\omega+3 \gamma)(\omega+4 \gamma)}}{3 \omega+10 \gamma}\left(\frac{3 \omega+10 \gamma}{\omega+2 \gamma}\right)^{-n}, & n \geq 1,\end{cases}
$$

and

$$
\eta_{n}^{(0)}= \begin{cases}\frac{2 \sqrt{\gamma(\omega+4 \gamma)}}{\omega+2 \gamma}\left(\frac{\omega+6 \gamma}{\omega+2 \gamma}\right)^{n}, & n \leq-2, \\ \frac{2 \sqrt{\gamma(\omega+4 \gamma)}}{\omega+6 \gamma}, & n=-1, \\ -\frac{2 \sqrt{\gamma(\omega+4 \gamma)}}{\omega+6 \gamma}, & n=0, \\ -\frac{2 \sqrt{\gamma(\omega+4 \gamma)}}{\omega+6 \gamma}\left(\frac{\omega+6 \gamma}{\omega+2 \gamma}\right)^{-n}, & n \geq 1 .\end{cases}
$$

It is clear from Eq. (28) that when $C>0$ the eigenvalue $\Omega_{E, 2}$ is always less than $\Omega_{L}$. As $C$ is increased, both $\Omega_{E, 1}$ and $\Omega_{E, 2}$ are decreased to zero but $\Omega_{E, 2}>\Omega_{E, 1}$ (note that $\Omega_{E, 2}$ is decreased more slowly than $\Omega_{E, 1}$ ). Therefore, it is concluded that the instability may occur due to $\Omega_{E, 1}$ becoming negative as illustrated in Fig. 1 . The critical value of $C$ can be found by equating $\Omega_{E, 1}=0$ as

$$
C_{c r}=\frac{\gamma(3 \omega+10 \gamma)(\omega+\gamma)}{(\omega+4 \gamma)^{2}},
$$

which approximate the onset of instability.

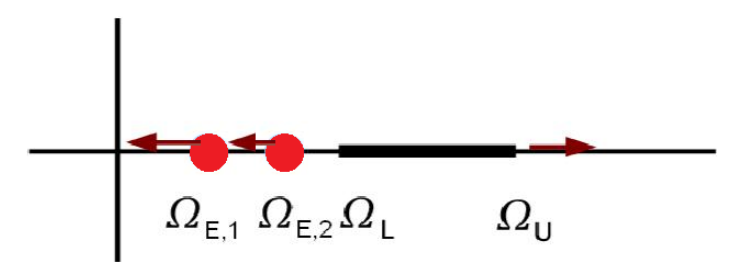

FIG. 1. A sketch of the dynamics of the eigenvalues and the continuous spectrum of a stable intersite dark soliton in the $(\operatorname{Re}(\Omega), \operatorname{Im}(\Omega)$ )-plane. The arrows indicate the direction of movement as the coupling constant $C$ increases. Note that a soliton is unstable if there is some $\Omega$ with either $\Omega<0$ or $\operatorname{Im}(\Omega) \neq 0$ 


\section{Comparison with numerical computations}

In this section, the numerical results are compared with the analytical calculation for previous section. The steady-state equation (2) is solved numerically by using Newton-Rapson method. Stability of this numerical solution is analyzed by solving the eigenvalue problem (4). $\omega$ is taken 0.9 in all the examples.

The comparisons between analytical predictions for the critical eigenvalues, given by equations (27)-(28) and corresponding numerical results are presented in Fig. 2. One should not be surprised by the appearance of the branching curves in the figure as they reveal the bifurcation of the critical eigenvalues from the inner edge of the continuous spectrum as soon as $C$ is turned on from which they then split into two distinct eigenvalues. It is seen that the approximations given by Eqs. (27)-(28) are in good agreement with the numerics. The range of validity of these approximations is wider for the upper branches in each branching curve.

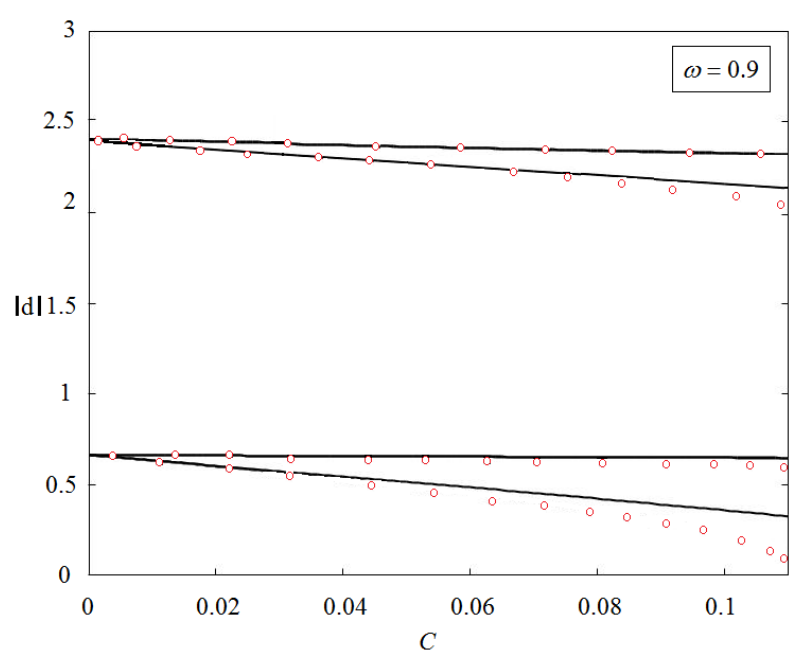

FIG. 2. Comparisons between the critical eigenvalue of intersite dark solitons obtained numerically (solid lines) and analytically (red dots) for two values of $\gamma$. The upper branching curves correspond to $\gamma=0.8$ while the lower ones to $\gamma=0.1$. The analytical approximations for the lower-upper branches in each branching curve are given by Eqs. (27)-(28)

The eigenvalue structures and corresponding soliton profiles of two values of $\gamma=0.1,0.8$ are plotted in Fig. 3 . For $\gamma=0.1$, when $C=0.04$ the eigenvalues $d$ lie in the gap between the two parts of the continuous spectrum, therefore no instability is obtained (Fig. 3(a)). For $\gamma=0.1$, when $C=0.5$ the instability is caused by a collision between one of the critical eigenvalues and its twin at the origin (Fig. 3(b)) (in Fig. 2, this eigen-value corresponds to the lower branch of the lower branching curves). If the value of $C$ is taken greater than 0.05 (taking $\gamma=0.1$ constant) the intersite dark solitons are remained always stable. It is interesting to note that the oscillatory instability can be fully suppressed by the presence of parametric driving, which has been appeared in onsite dark soliton [8]. As shown in the bottom panels of Fig. 3, there are values of the parameter $\gamma$ for which no instability-inducing collision ever occurs. The (in)stability region of this configuration as well as the analytical prediction for the onset of instability are summarised in Fig. 4. It is seen from the figure that for any $C$ and $\gamma>0.31$ an intersite dark soliton is remained always stable. By contrast, it is also seen that the intersite dark soliton is remaining always unstable for $\gamma<0$. The instability, in this case, is caused by the fact that the lower band of the continuous spectrum $\left(d=\sqrt{\Omega_{L}}\right)$ lies on the imaginary axis.

\section{Conclusions}

In this paper, analytical and numerical calculations are performed to determine the existence and stability of intersite dark discrete solitons in the parametrically driven discrete nonlinear Schrödinger equation. It has been shown that the presence of a parametric driving can stabilize intersite dark discrete solitons. An interval has been found in $\gamma$ for which a discrete dark soliton is stable for any value of the coupling constant, i.e., a parametric driving can suppress oscillatory instabilities. Stability windows for all the fundamental solitons have been presented and approximations using perturbation theory have been derived to accompany the numerical results. 

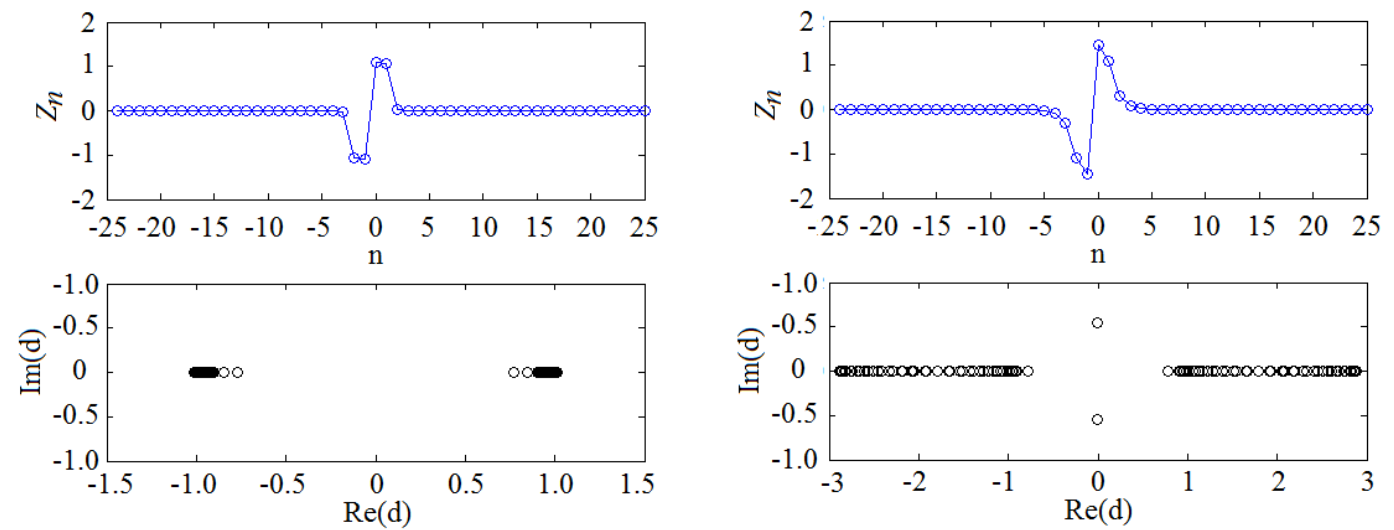

(a)
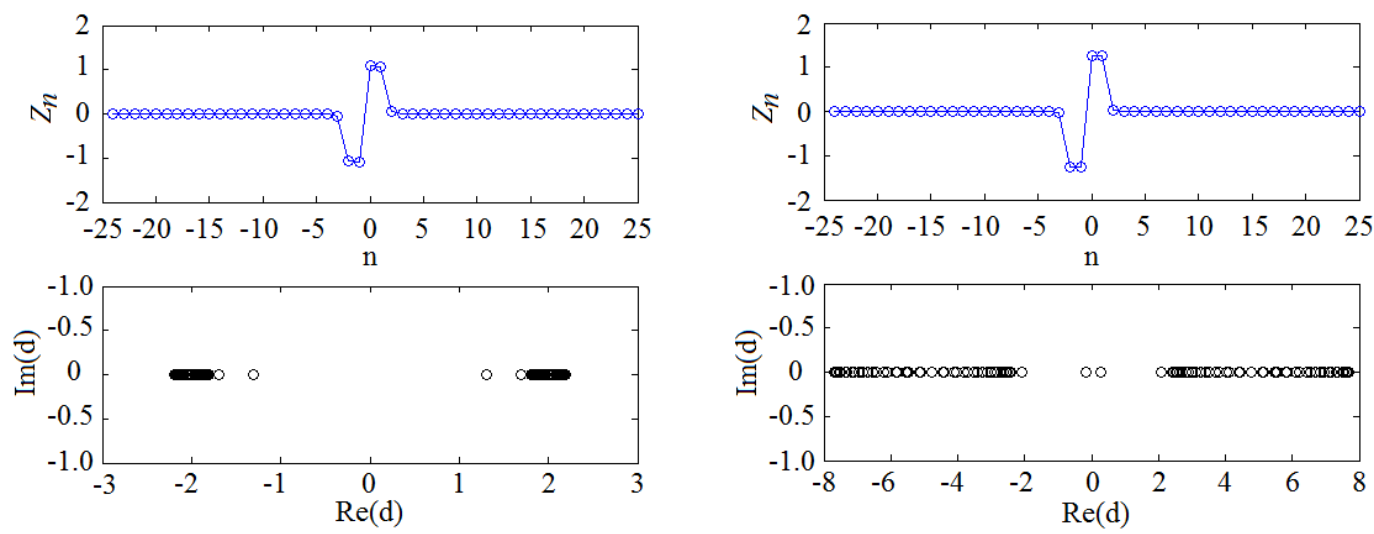

(c)

(d)

FIG. 3. The eigenvalue structures and soliton profiles of intersite dark solitons with parameter values as indicated in the caption for each panel. $\gamma=0.1, C=0.04$ (a); $\gamma=0.1, C=0.5$ (b); $\gamma=0.8, C=0.5(\mathrm{c}) ; \gamma=0.8, C=1.7$ (d)

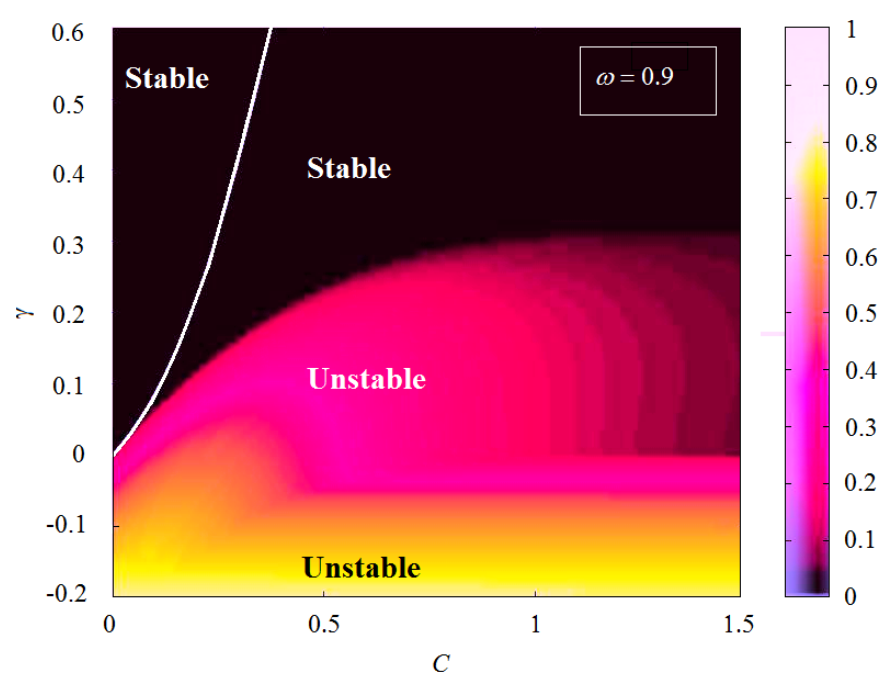

FIG. 4. The (in)stability region of intersite dark solitons in $(C, \gamma)$-space. For each value of $C$ and $\gamma$, the corresponding colour indicates the maximum value of $|\operatorname{Im}(d)|$ (over all eigenvalues $d$ ) for the steady-state solution at that point. Stability is therefore indicated by the region in which $\operatorname{Im}(d)=0$, namely the black region. Analytical approximation of Eq. (31) is given by white line. 


\section{References}

[1] Kevrekidis P.G. The Discrete Nonlinear Schr"odinger Equation: Mathematical Analysis, Numerical Computations and Physical Perspectives. Berlin, Springer, 2009.

[2] Lederer F., Stegeman G.I., et al. Discrete solitons in optics. Phys. Rep., 2008, 463, P. 1.

[3] Scott A. Encyclopedia of Nonlinear Science. New York and London, Routledge, 2005.

[4] Christinsen P.L., Scott A.C. Devydon's Soliton revisited, Self-tarpping of vibrational energy in protein. Denmark, Springer Science, 1989.

[5] Christodoulides D.N., Joseph R.I. Discrete self-focusing in nonlinear arrays of coupled waveguides. Optics Letters, 1988, 13 (9), P. 794-796.

[6] Susanto H., Johansson M. discrete dark solitons with multiple holes. Phys. Rev. E, 2005, 72, 016605(1-8).

[7] Syafwan M., Susanto H., Cox S.M. Discrete solitons in electromechanical resonators. Phys. Rev. E, 2010, 81 (2), 026207(1-14).

[8] Syafwan M. The existence and stability of solitons in discrete nonlinear Schrdinger equations. Ph.D. Thesis, University of Nottingham, 2012.

[9] Susanto H., Hoq Q.E., Kevrekidis P.G. Stability of discrete solitons in the presence of parametric driving type nonlinear Schrödinger lattices. Phys. Rev. E, 2006, 74, 067601(1-4).

[10] Swami O.P., Kumar V., Nagar A.K. Bright Solitons In A Parametrically Driven Discrete Nonlinear Schrödinger Equation. Int. J. Mod. Phys., 2013, 22, P. 570-575.

[11] Kaurov V.M., Kuklov A.B. Josephson vortex between two atomic Bose-Einstein condensates. Phys. Rev. A, 2005, 71, 011601(1-4).

[12] Kaurov V.M., Kuklov A.B. Atomic Josephson vortices. Phys. Rev. A, 2006, 73, 013627(1-8).

[13] Alexeeva N.V., Barashenkov I.V., Pelinovsky D.E. Dynamics of the parametrically driven NLS solitons beyond the onset of the oscillatory instability. Nonlinearity, 1999, 12, P. 103-140.

[14] Kivshar Yu.S. Bright and dark spatial solitons in non-Kerr media. Optical and Quantum Electronics, 1998, 30, P. 571-614.

[15] Hayata K., Koshiba M. Theory of stationary solitary waves generated by optical parametric interactions in the presence of Kerr-type nonlinearities and dissipations. J. Opt. Soc. Am. B, 1995, 12, P. 2288-2295.

[16] Baesens C., Kim S., MacKay R.S. localised modes on localised equilibria. Physica D, 1998, 133, P. 242. 